\title{
Imaging NEXAFS Spectroscopy of Cancerous Human Biological Tissue
}

\author{
E.L. Principe*, T. A. Cronin Jr., ${ }^{* *}$, S. McClary**, P.E. Sobol***, C. Jaye****, D. Fischer****, M. \\ Cronin*, K. Mitra* \\ *Florida Institute of Technology, Melbourne, FL, 32901 \\ **Cronin Skin Cancer Center, Melbourne, FL, 32901 \\ ***Synchrotron Research, Inc., Melbourne, FL, 32901 \\ ****National Institute of Standards \& Technology, Synchrotron Methods Group, Ceramics \\ Division, National Synchrotron Light Source, Upton, NY, 11973.
}

We report on the application of large area soft x-ray imaging near edge x-ray absorption fine structure (NEXAFS) spectroscopy of human biological tissue with a full field partial electron yield spectrometer. More commonly, biological tissue is characterized by NEXAFS in X-ray transmission mode, or Scanning Transmission X-ray Microscopy (STXM) [1,2]. While STXM provides higher lateral resolution, sample preparation is more arduous and the intensity of the focused beam causes significant radiation damage to soft tissue. The approach we demonstrate here employs a full field electron yield imaging NEXAFS spectrometer using far less radiation dose than STXM.

De-identified thin sections of dermal tissue containing basal cell carcinoma paired with control specimens from the same subject were prepared via cryo-microtome on glass slides. Sample preparation methods followed standard Mohs surgical procedure with exception that the sections were left unstained. All specimens were maintained in dry ice until transferred into the ultra high vacuum imaging spectrometer for analysis. Following NEXAFS analysis the samples were stained and optically imaged per the Mohs technique for direct comparison with the NEXAFS results.

Tissue samples on the glass slides were secured using copper tape and placed directly into a LARIAT MK I imaging NEXAFS spectrometer with no other sample preparation required. Cancerous versus non-cancerous tissue is differentiated in the images through spectroscopic analysis of the carbon and nitrogen near edges spectra. Differences observed are consistent with expected protein stoichiometric variations. One observation is that there are no analytical difficulties related to analysis of sensitive insulating tissue samples prepared without consideration of charge dissipation even in the presence of high intensity synchrotron soft x-ray radiation. This common method of tissue preparation is therefore suitable for imaging NEXAFS spectroscopy from a varied spectrum of biological tissues, opening new fields of investigation.

Hyperspectral imaging data was acquired in contiguous segments from $80-1100 \mathrm{eV}$ to acquire elemental surveys. Detail scans concentrated on the carbon, oxygen, nitrogen and iron chemistry. Chemical contrast of the nitrogen near edge spectrum is shown in Figure 1, depicting the distinction between the cellular structures derived from two of the spectral components displayed in Figure 2. We will present details of chemical contrast and the ability to discriminate healthy from diseased tissue in an effort to further characterize the chemistry of cancerous lesions. The lateral resolution of the LARIAT MKI is no better than 50um, while this is a useful resolution for biological specimens, higher lateral chemical image resolution is desirable. We will also briefly discuss progress in the production of a LARIAT MKII superconducting imaging NEXAFS spectrometer designed to achieve better than 5um lateral resolution over a $20 X 20 \mathrm{~mm}$ area and expected to come online late summer 2011. Application of imaging NEXAFS to biological materials is expected to benefit by the 600 fold improvement in areal resolution offered by the LARIAT MKII. 

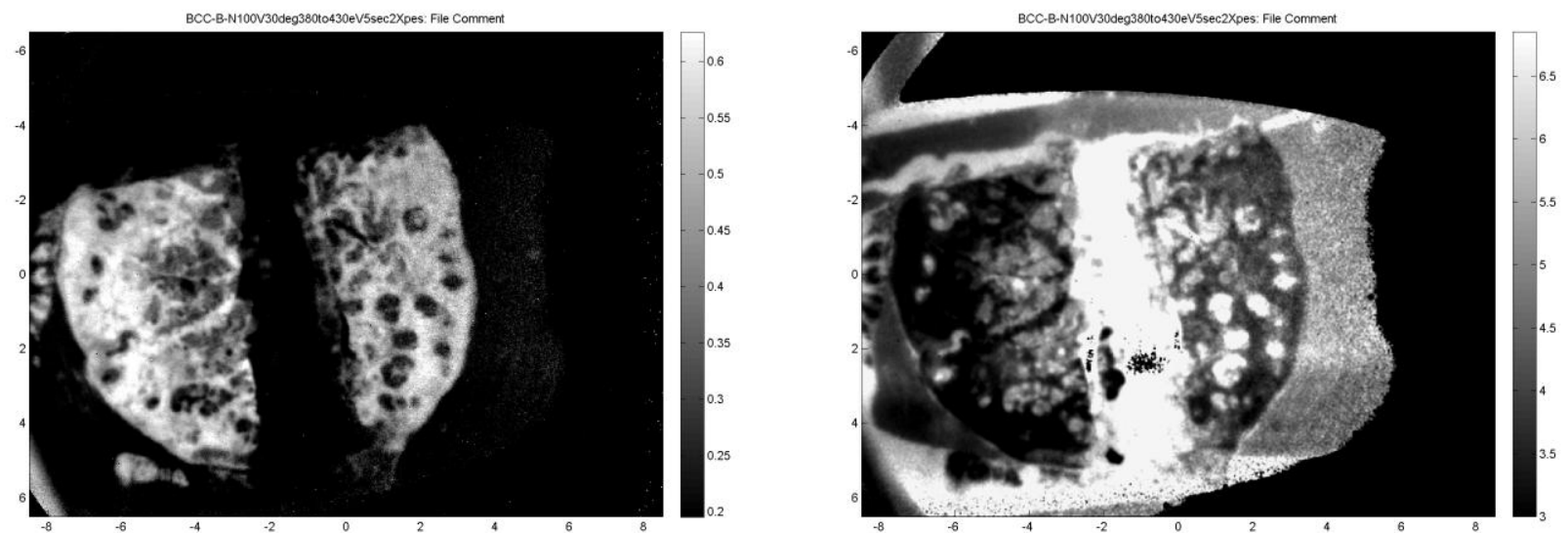

Figure 1. Imaging NEXAFS data from the phase I (left image) and phase II (right) image of nitrogen spectra obtained from a thin section of tissue containing basal cell carcinoma.

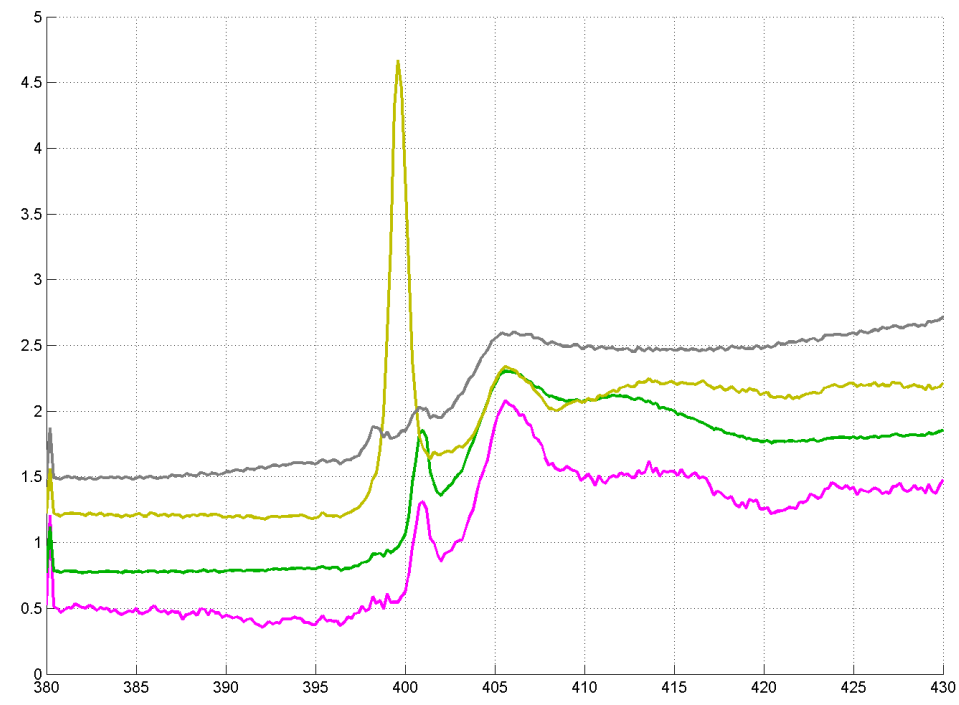

Figure 2 NEXAFS spectral data depicting four nitrogen chemistries observed in human skin tissue. Two of the components are mapped in Figure 1 above.

\section{References}

1. Kirz, J., Jacobsen, C., Howells, M., "Soft X-ray microscopes and their biological applications", Quarterly Reviews of Biophysics 28(1), pgs. 33-130, (1995).

2. Buckley, C J, Khaleque, N, Bellamy, S J, Robins, M, Zhang, X. "Mapping the Organic and Inorganic Components of Tissue Using NEXAFS”. Journal de physique. IV, Colloque : JP., 7(2), p.83, (1997). 